\title{
Fatores associados ao abuso de drogas em uma população clínica
}

\author{
Ana Carolina Peuker ${ }^{1}$ \\ Roberta Rosemberg \\ Silvia Mendes da Cunha \\ Lisiane Bizarro Araujo \\ Universidade Federal do Rio Grande do Sul, Porto Alegre-RS, Brasil
}

\begin{abstract}
Resumo: Foram investigadas as possíveis relações entre consumo de drogas, expectativas positivas quanto aos efeitos do álcool e sintomatologia depressiva e ansiosa. Participaram da pesquisa 40 indivíduos, com 26 anos em média, de ambos os sexos, atendidos em um serviço psicológico. Utilizou-se o Inventário de Expectativas e Crenças Pessoais Acerca do Álcool (IECPA), o Alcohol, Smoking and Substance Involvement Screening Test (ASSIST) e os Inventários de Beck de Ansiedade e Depressão. Constataram-se índices elevados de abuso de tabaco, álcool e observaram-se também expectativas positivas quanto aos efeitos do álcool. Tabagismo e ansiedade clinicamente significativa correlacionaram-se positivamente. Índices mais altos de expectativas positivas quanto ao consumo etílico estavam correlacionadas ao abuso de tabaco e álcool. Expectativas podem ser alteradas em programas preventivos, modificando-se padrões de consumo de risco. A associação entre sintomas psiquiátricos e uso de drogas deve ser considerada ao se estabelecerem programas preventivos e tratamentos.
\end{abstract}

Palavras-chave: distúrbios da ansiedade, depressão, droga (abuso).

\section{Factors associated with drug abuse in a clinical sample}

\begin{abstract}
Were investigated possible relationships between drug abuse and alcohol expectancies in a clinical population. Possible relationship between these variables and symptoms of depression and anxiety were explored. Participants $(n=40$, mean age 26) were both sexes individuals seen in a psychological service. Inventário de Expectativas e Crenças Pessoais acerca do Álcool (IECPA), Alcohol, Smoking and Substance Involvement Screening Test and Beck Anxiety and Depression Inventories were applied. There were high levels of prevalence of tobacco and alcohol abuse and positive alcohol expectancies. Male was related to higher alcohol consumption. There was a positive correlation between smoking and clinically significant anxiety symptoms. Moreover, it was found that more alcohol expectancies were related to tobacco and alcohol abuse. Alcohol expectancies can be modified in prevention programs, favoring a reduction in alcohol consumption and modification of consumption risk patterns. You should consider the association between psychiatric symptoms and drug use to establish prevention programs and treatments.
\end{abstract}

Keywords: anxiety disorders, major depression, drug abuse.

\section{Factores asociados con el abuso de drogas en una muestra clínica}

\begin{abstract}
Resumen: Fueron investigadas posibles relaciones entre el uso de drogas, las expectativas hacia el alcohol (EA), y sus relaciones con síntomas de depresión y ansiedad. Participaron 40 personas, con edad media de 26 años, de ambos sexos, atendidos en un servicio de psicología. Se utilizó el IECPA, el ASSIST y los inventarios Beck de Ansiedad y Depresión. Fueron constatados altos índices de abuso de tabaco y alcohol y expectativas positivas sobre los efectos del alcohol. El sexo masculino fue relacionado a un consumo mayor de alcohol. Se constató una correlación positiva entre el tabaquismo y ansiedad clínicamente significativa. Índices mas elevados de EA se relacionaron al abuso de tabaco y alcohol. EA podrán ser modificadas en programas de prevención, que favorezcan la reducción del consumo de riesgo. Deberá considerarse la asociación entre los síntomas psiquiátricos y el uso de drogas al establecer programas de prevención y tratamiento.
\end{abstract}

Palabras clave: trastornos de ansiedad, depresión, abuso de drogas.

Atualmente, o consumo de drogas é uma preocupação mundial, em função da sua alta incidência e dos riscos à saúde provenientes deste uso. A coocorrência de transtornos devido ao uso de substâncias psicoativas (SPAs) e transtornos mentais tem sido amplamente reconhecida. $\mathrm{O}$ abuso de SPAs é o transtorno mais frequente entre os portadores de transtornos mentais, logo é fundamental o diagnóstico adequado das patologias envolvidas (Ratto \& Cordeiro, 2004).

\footnotetext{
1 Endereço para correspondência:

Ana Carolina Peuker. Rua Ramiro Barcelos, 2600, sala 106. Universidade Federal do Rio Grande do Sul. Instituto de Psicologia, Laboratório de Psicologia Experimental, Neurociências e Comportamento (LPNeC). CEP 90.035-003. Porto Alegre- RS, Brasil.E-mail: acpeuker@terra.com.br.
}

Concomitante a isto, alguns estudos vêm relacionando o papel das expectativas acerca dos efeitos do uso da droga com a instalação de padrões de abuso e dependência. Além disso, outros estudos investigam o impacto das expectativas no prognóstico do tratamento.

A prevalência de comorbidades psiquiátricas em abusadores e dependentes de drogas é significativa, variando entre 21 e $65 \%$. A chance de indivíduos com transtorno por uso de substância apresentarem um ou mais transtornos mentais é quatro vezes maior do que pessoas que não usam substâncias. Dos transtornos possíveis, a depressão maior é o mais comum em dependentes químicos. É frequente que os transtornos relacionados ao consumo de álcool coexistam com outras doenças psiquiátricas (Alves, Kessler, \& Ratto, 2004; 
Kessler \& De Boni, 2006). Pacientes que fazem uso abusivo do álcool ou que dele são dependentes e que apresentam comorbidade psiquiátrica, principalmente aqueles com transtornos psiquiátricos graves, apresentam maiores taxas de suicídio e agressividade. Nestes casos, percebe-se também um número maior de detenção por atos ilegais, recaídas e internações. Além disso, este tipo de paciente utiliza mais os serviços de saúde e requer mais gastos com tratamento e maior período de hospitalização. Cabe lembrar ainda que estes pacientes, muitas vezes, não têm moradia (Alves e cols., 2004).

Considerando os estudos que relacionam abuso e dependência de substâncias com comorbidades psiquiátricas, entende-se que ansiedade e depressão se constituem como fatores de vulnerabilidade para o uso abusivo de substâncias (Calheiros, Oliveira, \& Andretta, 2006). Um terço dos alcoolistas apresenta um quadro significativo de ansiedade, e 50 a $67 \%$ dos alcoolistas e $80 \%$ dos dependentes de outras drogas possuem sintomas semelhantes ao transtorno do pânico, aos transtornos fóbicos ou ao transtorno de ansiedade generalizada (Bittencourt \& Oliveira, 2005; Edwards, Marshall, \& Cooks, 1999). Estima-se que de 23 a 70\% dos pacientes dependentes de álcool e outras substâncias sofram de transtornos ansiosos ou depressivos, comorbidades mais comumentemente associadas a esses pacientes (Alves e cols., 2004; Monteiro, 1997), sendo importante a realização de um diagnóstico preciso.

Tabagistas também apresentam transtornos de ansiedade, com prevalência de transtorno de ansiedade generalizada e fobia específica (Munaretti \& Terra, 2007). Portanto, é necessário considerar tanto os sintomas ansiosos, como também os sinais de depressão em tabagistas, uma vez que esses sintomas podem se relacionar com o prognóstico do tratamento, que visa à cessação do uso do cigarro bem como almeja menores taxas de recaída (Glassman, Covey, Stetner, \& Rivelli, 2001; Mello, Oliveira, \& Ferreira, 2006). Estudos empíricos revisados por Morissette, Tull, Gulliver, Kamholz e Zimering (2007) sugerem que a nicotina tem efeito ansiolítico e que o cigarro pode ser usado, então, como uma estratégia de enfrentamento dos sintomas ansiosos.

\section{Aspectos cognitivos e comportamentais associados ao consumo de SPAs}

Expectativas positivas e estados afetivos relacionados aos efeitos de SPAs podem exercer influências importantes no início e na manutenção do uso de substâncias psicoativas e na emissão de comportamentos relacionados a este consumo (Araujo \& Gomes, 1998; Fromme \& D'Amico, 2000; Goldman, 1999). Indivíduos que mantêm expectativas positivas quanto aos efeitos do uso de drogas têm maior probabilidade de tornarem-se dependentes delas. Mesmo antes de fazerem alguma experimentação, os indivíduos já vão formando crenças, ideias e expectativas, antecipando os possíveis efeitos do uso de drogas, pois as expectativas desenvolvem-se a partir de modelos parentais e do grupo de pares, além de experiências diretas e indiretas com bebidas alcoólicas (Christiansen, Smith, Roehling, \& Goldman, 1989; Donovan, \& Marlatt, 1980; Peuker, Fogaça, \& Bizarro, 2006). Muitas vezes, os indivíduos antecipam os efeitos positivos imediatos do comportamento, com base em uma experiência prévia (Marlatt \& Gordon, 1993; Pedroso, Oliveira, Araújo, Castro, \& Melo, 2006; Peuker e cols., 2006).

Os aspectos culturais e ambientais em conjunto com as crenças cognitivas e as expectativas pessoais sobre os resultados esperados do uso de uma determinada substância são fatores preditivos do uso abusivo. O processo de tomada de decisão envolvendo o consumo de SPAs é influenciado pela expectativa de que o uso trará consequências desejáveis, tais como a diminuição da tensão e a facilitação na interação social (Gaffney, Thorpe, Young, Collet, \& Occhipint, 1998; Marlatt \& Gordon, 1993; Pedroso e cols., 2006). Considerar tais expectativas é importante no tratamento e no prognóstico de indivíduos com transtornos relacionados ao uso de drogas (Pedroso e cols., 2006).

\section{Desafios no diagnóstico e no tratamento do abuso ou dependência de SPAs}

Evidencia-se a crescente necessidade de um melhor treinamento dos profissionais de saúde para identificação e manejo de pacientes que apresentam abuso e dependência de álcool e tabaco e/ou dependentes químicos. Existem dificuldades na abordagem terapêutica destes pacientes, os quais, geralmente, não encontram locais adequados para tratamento. Nesse sentido, profissionais da saúde têm um papel decisivo na detecção precoce do abuso e da dependência de substâncias psicoativas, sendo os instrumentos de avaliação importantes dentro deste contexto (Alves e cols., 2004).

Os profissionais da saúde tendem a ter dificuldades em realizar um diagnóstico preciso em relação aos transtornos mentais relacionados ao uso de SPAs, uma vez que há presença frequente de comorbidades nestas psicopatologias. Instrumentos padronizados têm sido utilizados para auxiliar a avaliação dos critérios diagnósticos e o estabelecimento do plano terapêutico. O uso de instrumentos de avaliação pode, também, ser útil na triagem de pacientes em instituições de atenção à saúde (Henrique, De Micheli, Lacerda, Lacerda, \& Formigoni, 2004). É importante que estratégias de avaliação adequadas possam ser empregadas com intuito de favorecer a intervenção terapêutica, uma vez que se observam índices elevados de comorbidades entre abuso e dependência de substâncias psicoativas e outros transtornos psiquiátricos, principalmente transtornos ansiosos e depressão (Castel, Rush, Urbanoski, \& Toneatto, 2006).

Neste contexto, este estudo foi conduzido com o objetivo geral de caracterizar o perfil de consumo (uso ocasional, abuso ou dependência) de SPAs de uma amostra clínica. Também foram investigadas as possíveis relações entre 
consumo de SPAs, expectativas positivas quanto aos efeitos do álcool e sintomatologia depressiva e ansiosa.

\section{Método}

\section{Participantes}

Por conveniência, a seleção da amostra foi não-probabilística, método de amostragem comumentemente utilizado em ciências do comportamento humano (Cozby, 2003). Foram convidadas a participar do estudo aproximadamente 80 pessoas. Deste total, apenas 47 (59\%) consentiram em colaborar. Além disso, sete protocolos (15\%) foram entregues com falta de informações essenciais para a análise (por exemplo, questionários rasurados ou preenchidos inadequadamente). Sendo assim, a pesquisa contou com 40 participantes (dos quais 27 eram mulheres) com idade média de 26 anos. Os participantes buscaram atendimento psicológico e/ou psiquiátrico em uma clínica de atendimento médico e psicológico não especializada no tratamento de dependência química. Do total de participantes, 19 (47,5\%) eram solteiros, $14(35 \%)$ eram casados, um (2,5\%) vivia em união estável e seis (15\%) não responderam qual era seu estado civil.

\section{Instrumentos}

ASSIST (Alcohol, Smoking and Substance Involvement Screening Test)

O ASSIST é um questionário estruturado, contendo oito questões sobre o uso de nove classes de substâncias psicoativas (tabaco, álcool, maconha, cocaína, estimulantes, sedativos, inalantes, alucinógenos e opiáceos). Cada resposta corresponde a um escore, que varia de 0 a 4 , sendo que a soma pode variar de 0 a 20. Este instrumento (fruto de um projeto multicêntrico) foi desenvolvido e coordenado pela Organização Mundial da Saúde (OMS) e posteriormente traduzido e validado no Brasil. No estudo da validação da versão brasileira, o ASSIST apresentou boa sensibilidade e especificidade na detecção de uso abusivo/dependência de álcool, maconha e cocaína. A confiabilidade do instrumento foi adequada (alfa de Cronbach de 0,80 para álcool, 0,79 para maconha e 0,81 para cocaína). Segundo os autores da versão brasileira, as propriedades psicométricas do ASSIST apresentam-se satisfatórias, possibilitando sua aplicação a pacientes de serviços de atenção primária/secundária à saúde (Henrique e cols., 2004).

\section{Inventário de Expectativas e Crenças Pessoais acerca do Álcool (IECPA)}

Este instrumento, desenvolvido por Gouveia, Ramalheira, Robalo, Borges e Almeida (1996), teve sua versão brasileira adaptada. Trata-se de uma medida escalar, do tipo Likert, com 61 itens. Cada item consiste numa afirmação, que envolve expectativas e crenças a respeito dos efeitos do álcool, distribuídas em cinco fatores. Estes integram o IECPA e incluem efeitos globais positivos (beber me deixa mais corajoso) e facilitação das interações sociais (o álcool me torna mais alegre e simpático), diminuição e/ou fuga emoções negativas (o álcool me faz esquecer os problemas da vida), ativação e prazer sexual (tenho mais prazer sexual após ter bebido), efeitos positivos na atividade e humor (quando bebo aprecio melhor as coisas boas da vida) e na avaliação de si mesmo (quando bebo fico menos nervoso). Para cada item, são apresentadas cinco alternativas de resposta: não concordo, concordo pouco, concordo moderadamente, concordo muito e concordo muitíssimo. Cada opção de reposta recebe um escore de um a cinco. O resultado final varia de zero a 305 pontos.

A pressuposição subjacente é a de que maiores escores no IECPA caracterizam participantes com expectativas positivas mais altas e, portanto, maior vulnerabilidade ao alcoolismo. Se o indivíduo é da população geral, o ponto de corte é de 121,82. Ou seja, aqueles que apresentam escore total de 122 ou mais têm probabilidade de já serem ou virem a ser dependentes de álcool. Quanto às propriedades psicométricas, a versão brasileira do IECPA possui um excelente índice de consistência interna (alfa de Cronbach=0,984). O instrumento também apresentou estabilidade temporal, por meio do teste-reteste - coeficiente de correlação=0,865 (Gouveia e cols., 1996).

\section{Inventário Beck de Ansiedade (BAI)}

$\mathrm{O}$ inventário é composto de 21 itens, relacionados à presença de sintomas ansiosos. Para cada item, o sujeito deve escolher um dentre quatro níveis de ansiedade, ou seja, deve optar por um ponto numa escala do tipo Likert de quatro pontos. Estes variam de 0 a 3, demonstrando a evolução do grau de intensidade dos sintomas. A soma dos valores obtidos em cada item resulta em um escore total, que varia entre zero e 63 pontos. De acordo com cada escore total apresentado, há a indicação de um determinado diagnóstico: entre zero e 10 pontos, ausência de sintomas ansiosos; entre 11 e 19 pontos, ansiedade leve à moderada; entre 20 e 30 pontos, ansiedade moderada; entre 31 e 63 pontos, ansiedade grave. Cabe salientar que o escore de 21 pontos é considerado indicativo da existência de ansiedade clinicamente significativa. $\mathrm{O}$ instrumento apresenta boa consistência interna $(\alpha=0,92) \mathrm{e}$ confiabilidade do tipo teste e reteste (Beck, Epstein, Brown, \& Steer, 1988; Cunha, 2001).

\section{Inventário de Depressão de Beck (BDI)}

É uma escala de autorrelato para levantamento da intensidade de sintomas depressivos. É um instrumento estruturado, composto de 21 categorias de sintomas e atitudes, que descrevem manifestações comportamentais, cognitivas, afetivas e somáticas da depressão. Cada categoria contém quatro ou cinco alternativas, que expressam níveis de gravidade dos sintomas depressivos. Quanto à classificação da intensidade dos sintomas, a normatização da versão brasileira do BDI estabelece intensidade mínima (0-11), leve (12-19), moderada (2035) e grave (36-63) (Cunha, 2001; Oliveira, Wagner, Zambon, 
$\&$ Calheiros, 2006). Contudo, o escore de 21 pontos ou mais tem sido sugerido como indicativo da existência de depressão clinicamente significativa (Maluf, 2002).

O BDI apresenta 0,86 de índice de confiabilidade e $97 \%$ de concordância entre entrevistadores. Além disso, a confiabilidade diagnóstica desse instrumento pode ser confirmada por meio do procedimento de teste-reteste (Beck, Ward, Mendelson, Mock, \& Erbaugh, 1961). No Brasil, o BDI foi validado por Gorestein e Andrade (1996), com índices de consistência interna $(0,81)$ para a população geral e $(0,88)$ para a população clínica.

\section{Procedimentos de coleta de dados}

Os procedimentos para coleta de dados e a divulgação dos resultados do trabalho seguiram as diretrizes para a pesquisa com seres humanos estabelecidas pelo Conselho Federal de Psicologia - Resolução n. ${ }^{\circ} 16 / 2000$ - e pelo Conselho Nacional de Saúde (1996) - Resolução n. ${ }^{\circ}$ 196/96. Dessa forma, garantiu-se a proteção dos dados dos participantes da pesquisa.

Aqueles que aceitaram participar da pesquisa preencheram o Termo de Consentimento Livre e Esclarecido. Estabeleceu-se a idade mínima de 18 anos como critério de inclusão no estudo, conforme protocolo aprovado pelo Comitê de Ética em Pesquisa do Instituto de Psicologia da UFRGS.

Foram convidados a participar do estudo todos os pacientes que se encontravam em tratamento psicológico e/ou psiquiátrico, ou em processo de triagem, no período de março a abril de 2008. A coleta de dados foi realizada por duas psicólogas capacitadas para o preenchimento dos instrumentos e aptas para os esclarecimentos a respeito do estudo.

Para garantir o sigilo e a confidencialidade acerca da identidade dos participantes, os instrumentos de coleta foram distribuídos em envelopes codificados com números, sem identificação nominal. Os envelopes eram entregues fechados para que os participantes respondessem às questões formuladas. Foram esclarecidas dúvidas iniciais a respeito do preenchimento dos instrumentos e dos objetivos e procedimentos da pesquisa. Logo após, os pacientes eram encaminhados a uma sala reservada, onde respondiam individualmente aos questionários. Depois disso, os envelopes, devidamente lacrados, eram devolvidos pelos participantes à equipe de pesquisa. $\mathrm{O}$ tempo médio de preenchimento foi em torno de 35 minutos.

Parte dos participantes estava sob atendimento psicológico e/ou psiquiátrico, conduzidos por profissionais que seguiam distintas orientações teóricas. Sendo assim, foram incluídos na amostra tanto pacientes que estavam em psicoterapia de orientação analítica como também aqueles que estavam em atendimento sob orientação cognitivocomportamental. Cabe salientar que tais pacientes se encontravam em fases distintas do tratamento. Desta maneira, a amostra foi composta tanto por pacientes que ainda estavam em processo de avaliação inicial (triagem) para posterior encaminhamento ao serviço psiquiátrico e/ou psicológico, quanto por pacientes que já estavam em atendimento psicológico e/ou psiquiátrico.

\section{Procedimento de análise de dados}

Primeiramente, os dados foram analisados considerando os procedimentos de estatística descritiva para análise das variáveis estudadas em termos de distribuição de frequências, escores, médias e desvio padrão. No BAI e no BDI, adotou-se um ponto de corte de 21, indicativo de ansiedade e depressão, respectivamente, clinicamente significativa. Analisou-se a correlação entre os fatores de risco para uso abusivo de substâncias psicoativas, entre os quais: altas expectativas positivas em relação ao álcool, sintomatologia indicativa de depressão e ansiedade e o desfecho (abuso ou dependência de substâncias psicoativas) por meio do teste estatístico exato de Fisher. Em todas as análises realizadas, adotou-se um nível de significância de 5\% e empregou-se o pacote estatístico SPSS (Statistical Package for the Social Sciences) versão 14.

\section{Resultados}

\section{Perfil do consumo de SPA's}

Os resultados obtidos a partir do ASSIST revelaram que $70 \%(n=28)$ dos participantes faziam uso ocasional de tabaco, 22,5\% (n=9), uso abusivo e 7,5\% (n=3) não responderam. O uso ocasional de tabaco foi relatado por $77,8 \%(n=21)$ das mulheres, e o uso abusivo, por $22,2 \%(n=6)$. Entre os homens, $66,7 \%(n=6)$ referiam uso ocasional e $33,3 \%(n=3)$, uso abusivo. Em relação ao uso de tabaco $(\mathrm{p}=0,660)$, não foi encontrado diferença entre os sexos.

No que concerne ao uso de álcool, $57,5 \%(n=23)$ dos participantes referiram uso ocasional e $35 \%(n=14)$, uso abusivo e 7,5\% $(n=3)$ não responderam. Entre as mulheres, encontrou-se um percentual de $74,1 \%(n=20)$ de uso ocasional e $25,9 \%(n=7)$ de uso abusivo do álcool. Para o sexo masculino, observou-se que $22,2 \%(n=2)$ revelaram uso ocasional e 77,8\% (n=7), uso abusivo. Foi constatada diferença entre os sexos em relação ao uso de álcool $(\mathrm{p}=0,014)$.

Quanto às outras classes de drogas investigadas através do ASSIST (maconha, cocaína, estimulantes, inalantes, hipnóticos, alucinógenos e opióides), não foi identificado uso abusivo nem dependência na amostra. Em função disso, as análises de correlação com sintomas de ansiedade, depressão e expectativas quanto aos efeitos destas outras drogas não foram realizadas.

\section{Sintomas de ansiedade e depressão}

Observou-se que $37,5 \%(n=15)$ dos participantes não obtiveram escores clinicamente significativos de ansiedade. Já $42,5 \%(n=17)$ referiram escores de sintomatologia ansiosa clinicamente significativa. Houve $20 \%(n=8)$ de não respondentes.

No BDI, constatou-se que $75 \%(n=30)$ dos participantes 
não evidenciaram sintomatologia indicativa de depressão clinicamente significativa, enquanto que um percentual de $7,5 \%(n=3)$ apresentou escores compatíveis com sintomatologia depressiva clinicamente significativa e $17,5 \%(n=7)$ não responderam.

\section{Expectativas positivas quanto aos efeitos do álcool}

No IECPA, constatou-se que 50\% $(n=20)$ dos participantes obtiveram escores menores do que 122 . Ou seja, por este critério, não evidenciaram risco de serem ou de se tornarem dependentes de álcool. Mas, 30\% $(n=12)$ revelaram tal risco, isto é, apresentaram escores maiores ou iguais a 122. Ainda, 20\% $(\mathrm{n}=8)$ não responderam ao inventário.

\section{Fatores associados ao abuso e/ou à dependência de tabaco e álcool}

Nesta análise, foram incluídos apenas o tabaco e o álcool, visto que apenas para estas substâncias foi identificado uso mais que ocasional.

\section{Análise dos fatores associados ao abuso e/ou à dependência de tabaco: sintomas de ansiedade, depressão e expectativas em relação aos efeitos do álcool}

Em relação ao uso de tabaco, constatou-se, através do BAI e dos resultados do ASSIST, que, dentre os participantes sem sintomatologia ansiosa $(n=15), 100 \%$ referiram fumar ocasionalmente. Já entre aqueles com sintomas indicativos de ansiedade $(n=15), 60 \%(n=9)$ revelaram fazer uso ocasional e $40 \%(n=6)$, uso abusivo desta substância. Constatouse, através de análise estatística, uma diferença significativa entre os grupos com e sem sintomatologia de ansiedade em relação ao uso de tabaco $(\mathrm{p}=0,016)$. Considerando os escores do BDI, não foram encontradas diferenças significativas entre pacientes com e sem sintomatologia depressiva e uso de tabaco.

A partir dos resultados do IECPA e do ASSIST em relação ao tabaco, constatou-se que, entre os participantes com expectativas positivas mais baixas em relação ao álcool $(n=20), 95 \%(n=19)$ fumam ocasionalmente e $5 \%(n=1)$ fazem uso abusivo desta substância. Já entre os participantes com expectativas positivas mais altas $(n=11), 45,5 \%(n=5)$ faziam uso ocasional do tabaco e $54,5 \%(n=6)$ relataram fazer uso abusivo. Verificou-se que os dois grupos diferiram entre si quanto ao uso do tabaco $(\mathrm{p}=0,004)$.

\section{Análise dos fatores associados ao abuso e/ou à dependência de álcool: sintomas de ansiedade, depressão e expectativas em relação aos efeitos do álcool}

Em relação ao uso de álcool, os escores do BAI e os resultados do ASSIST não indicaram diferença significativa entre os grupos com e sem sintomatologia ansiosa. Considerando o BDI, também não foram encontradas diferenças entre pacientes com e sem sintomatologia depressiva e uso de álcool.

Através do IECPA, evidenciou-se que, entre os participantes com expectativas positivas em relação ao álcool altas $(n=11), 36,4 \%(n=4)$ faziam uso ocasional e $63,6 \%(n=7)$, uso abusivo de álcool. Participantes com expectativas positivas baixas $(n=20), 80 \%(n=16)$ referiram fazer uso ocasional e $20 \%(n=4)$, uso abusivo. No que diz respeito ao uso/abuso do álcool, detectou-se uma diferença estatisticamente significativa entre os grupos com baixas expectativas (escores $<122$ ) e altas expectativas em relação aos efeitos do álcool (escores $\geq 122$ ). Ou seja, observou-se que indivíduos com maiores expectativas positivas acerca dos efeitos do álcool faziam maior uso desta substância $(\mathrm{p}=0,023)$.

\section{Discussão}

No que se refere ao perfil de consumo de SPAs, foi identificado padrão de uso abusivo de tabaco em $22,5 \%(n=9)$ e padrão de uso abusivo de álcool em $35 \%(n=14)$ dos participantes. Estes podem ser considerados índices elevados de abuso destas substâncias. O II Levantamento Domiciliar Sobre o Uso de Drogas Psicotrópicas no Brasil viabilizou dados sobre o perfil de uso e abuso de SPAs no país. Tal levantamento permitiu identificar índices de $12,5 \%$ de dependência de álcool e 10,11\% de tabagismo para a população brasileira. Ademais, este mesmo levantamento obteve dados referentes à região Sul, onde as análises revelaram $9 \%$ de dependência de álcool e 10,7\% de tabagismo (Carlini e cols., 2006).

Embora a prevalência do consumo de tabaco na população geral seja alta, esta taxa cresce sensivelmente entre pacientes psiquiátricos (Malbergier \& Oliveira Jr., 2005). Em um estudo prévio realizado com pacientes atendidos em um ambulatório de psiquiatria, na mesma cidade que a presente investigação aconteceu, o percentual de tabagistas representou $21,43 \%$ da amostra, sendo que a maior parte deles possuía nível leve de dependência de nicotina (Munaretti \& Terra, 2007).

No que concerne ao tabaco, pôde-se constatar que $70 \%$ $(n=28)$ dos participantes faziam uso ocasional e que $22,5 \%$ $(n=9)$ faziam uso abusivo desta substância. Entre os participantes com sintomatologia ansiosa clinicamente significativa (escores $\geq 21$ ), observou-se que $40 \%$ faziam uso abusivo desta droga. Já, entre os participantes sem sintomatologia ansiosa, detectou-se apenas uso ocasional. Este achado corrobora a literatura revisada, uma vez que a frequência de tabagismo entre pacientes com transtorno de ansiedade é alta (Calheiros e cols., 2006; Malbergier \& Oliveira Jr., 2005; Munaretti \& Terra, 2007). Observa-se uma importante associação do tabagismo com transtornos ansiosos. Por isso, a classe de transtornos ansiosos também deve ser foco de atenção no tratamento desses pacientes. Para relacionar a incidência conjunta entre tabagismo e ansiedade, deve-se considerar o diagnóstico específico de transtorno de ansiedade (Rondina, Gorayeb, \& Botelho, 2003).

Os transtornos de ansiedade têm prevalência significativa na população. Considerando que estes repercutem negativamente nas relações pessoais, nos estados emocionais e que geram encargos sociais, é fundamental identificar sintomas ansiosos que possam indicar a presença de algum transtorno 
de ansiedade, visto que eles tendem a ser crônicos se não tratados (Munaretti \& Terra, 2007). Além disso, considerando-se que o tabagismo está relacionado à ocorrência de transtornos de ansiedade, é importante que estes possam ser identificados e que o tratamento adequado possa ser realizado com intuito de prevenir o uso e a instalação da dependência do tabaco (Malbergier \& Oliveira Jr., 2005).

Apesar dos índices de associação elevados encontrados entre uso abusivo do tabaco e sintomatologia ansiosa clinicamente significativa, não é possível estabelecer-se relações de causalidade. Ou seja, por se tratar de um estudo transversal e não um estudo de seguimento, não é possível inferirmos se foi a sintomatologia ansiosa, ao longo do tempo, que contribuiu para a experimentação e a instalação do tabagismo. Por outro lado, também, é possível que indivíduos tabagistas se configurem como um grupo de risco para o desenvolvimento de sintomatologia ansiosa, principalmente nos períodos de abstinência e tentativas de cessação do uso do tabaco (Morissette e cols., 2007).

Apesar de a literatura indicar uma forte evidência de associação entre tabagismo e transtornos depressivos, isto não foi observado entre os participantes deste estudo. Nos tabagistas com histórico de transtornos depressivos, a literatura relata, por exemplo, que a cessação do comportamento de fumar constitui fator de risco para a manutenção do quadro clínico ou reincidência de depressão. Neste sentido, o uso de tabaco pode funcionar como um tipo de automedicação, aliviando os sentimentos de tristeza ou humor negativo (Calheiros e cols., 2006).

Observou-se que os participantes que mantinham expectativas positivas altas acerca do álcool faziam mais uso do tabaco. Entre pacientes com expectativas mais altas em relação ao uso de álcool havia um maior índice de uso abusivo de álcool $(63,6 \% ; n=7)$. Este dado está de acordo com a literatura, que sugere uma forte relação entre tabagismo e transtornos de abuso e/ou dependência de álcool. A prevalência de alcoolismo em tabagistas é de aproximadamente dez a 14 vezes maior, em comparação aos não-tabagistas.

Quanto ao uso de álcool, 57,5\% $(n=23)$ dos participantes referiram fazer uso ocasional e $35 \%(n=14)$ destes referiram uso abusivo. Constatou-se maior abuso de álcool entre os homens $(25,9 \%, n=7)$ do que entre as mulheres $(22,2 \%$, $n=2)$. Este resultado está em consonância com estudos prévios, que sugerem que a prevalência do alcoolismo entre os homens é maior do que a encontrada entre as mulheres (Blume, Green, Joanning, \& Quinn 1994; Grant, 1997). Apesar disso, o abuso ou a dependência desta substância repercute negativamente sobre a saúde física, psíquica e social das mulheres. Há evidência de que mulheres que consumiam acima de três doses diárias de bebidas alcoólicas apresentavam risco mais elevado para suicídio e acidentes fatais (Ross, Glaser, \& Stiasny, 1998). Embora o consumo de álcool seja menor entre as mulheres, os prejuízos decorrentes deste consumo podem ser maiores para elas do que para os homens (Bongers, Oers, Goor, \& Garretsen 1997).
O fato de não ter sido detectada diferença estatisticamente relevante entre participantes com e sem ansiedade, com e sem depressão em relação ao uso de álcool pode ser atribuído ao ponto de corte adotado no BAI $(\geq 21)$ e no BDI $(\geq 21)$, que indicava apenas níveis de ansiedade e depressão mais elevados, ou seja, clinicamente significativos. Isso não quer dizer que não exista associação entre sintomas de ansiedade e depressão mais leves e moderados com o uso ocasional e abusivo de álcool. O tamanho restrito da amostra também pode ter comprometido a identificação desta possível associação, pois há indícios consistentes de que transtornos ansiosos ou depressivos estejam entre as comorbidades mais comuns associadas a pacientes dependentes de álcool e outras substâncias (Alves e cols., 2004; Calheiros e cols., 2006). Além disso, os participantes estavam sob acompanhamento psicológico e/ou psiquiátrico. Há evidências de que a psicoterapia aliada ao tratamento medicamentoso pode contribuir para uma melhoria significativa nos sintomas do humor e de ansiedade, além de diminuir o consumo de álcool e as taxas de recaída (Alves e cols., 2004).

Neste estudo, adotou-se um ponto de corte de 122 no IECPA. Ou seja, aqueles participantes que apresentaram escore 122 ou mais teriam maior probabilidade de serem ou virem a se tornar dependentes de álcool. De acordo com isso, observou-se, entre aqueles indivíduos que apresentaram expectativas positivas mais altas em relação ao álcool, percentuais de uso ocasional $(36,4 \%)$ e abusivo $(63,6 \%)$ maiores do que aqueles com baixas expectativas positivas ( $80 \%$ uso ocasional e $20 \%$, abusivo). Este dado está alinhado com a literatura que indica que indivíduos com expectativas mais altas em relação aos efeitos do uso de álcool têm maior probabilidade de serem ou virem a se tornar dependentes de álcool (Gouveia e cols., 1996).

Constatou-se que $20 \%(n=8)$ dos participantes não responderam ao IECPA. Este fato pode estar relacionado à extensão e complexidade do instrumento (61 itens), que exigia certo nível cognitivo para compreender as questões propostas e respondê-las. Por se tratar de uma amostra clínica, na qual se infere uma maior prevalência de problemas psiquiátricos, possivelmente limitações cognitivas associadas a tais condições psicopatológicas possam ter interferido no adequado preenchimento do referido instrumento.

Pessoas com expectativas positivas em relação aos efeitos do álcool (por exemplo, expectativa de que o álcool aumentaria a confiança em situações sociais ou aliviaria a tensão) têm maior propensão a reportarem problemas sócioemocionais mais graves, como depressão, ansiedade, dificuldade de relacionamento com familiares ou com outras pessoas e sentimentos negativos sobre si mesmos (O'Hare $\&$ Scherrer, 1997). Ademais, observa-se que bebedores frequentes mantêm expectativas positivas, que geralmente estão incorretas. Sugere-se que bebedores-problema têm mais expectativas positivas sobre os efeitos imediatos do álcool que expectativas acerca de suas consequências negativas a longo prazo (Lewis \& O’Neill, 2000; Peuker e cols., 2006). 


\section{Considerações finais}

As particularidades referentes ao abuso e à dependência de substâncias psicoativas devem ser investigadas em amostras clínicas, tendo em vista o alto índice de morbidade e mortalidade associado a estes transtornos. Sabe-se, ainda, que as expectativas pessoais acerca do uso destas substâncias podem ser um fator de risco para o desenvolvimento de comportamentos relacionados ao uso abusivo e à dependência. Portanto, ressalta-se a importância de investigar tais expectativas e, a partir delas, ampliar a possibilidade de intervenções terapêuticas.

A revisão da literatura indicou que os transtornos de humor e ansiedade têm sido frequentemente associados à dependência química. Desta forma, estudos diagnósticos e de tratamento são importantes para melhor compreender este problema e assim buscar intervenções mais eficazes e métodos preventivos. A prevenção, além de trazer benefícios importantes aos indivíduos, reduz os gastos em saúde pública.

Os elevados índices de prevalência do uso abusivo de tabaco, álcool e altas expectativas positivas em relação ao consumo etílico encontrados neste estudo devem ser analisados considerando-se que a amostra investigada é clínica. Estes achados confirmam a literatura que indica que a prevalência do uso de drogas em populações portadoras de transtornos psiquiátricos é mais elevada do que na população geral. Sugere-se que tais associações podem ser mediadas por variáveis genéticas e neurobiológicas (Messas \& Vallada, 2004; Munaretti \& Terra, 2007; Rondina e cols., 2003).

A identificação das expectativas positivas em relação aos efeitos do álcool pode contribuir para que sejam desenvolvidos programas preventivos adequados à população investigada. As expectativas podem ser modificadas em programas de prevenção. Dessa maneira, favorecendo-se a redução do consumo de álcool, modificar-se-ão os padrões de consumo de risco (Peuker e cols., 2006). Também se deve considerar a associação entre transtornos de ansiedade, humor e uso de drogas ao se estabelecer programas de natureza preventiva e tratamentos.

Os resultados desta investigação devem ser interpretados com cautela em função do número reduzido de participantes, da não-aleatoriedade da amostra e do predomínio de mulheres. Futuras investigações devem considerar, além da inclusão de um número maior de homens, uma descrição detalhada da população clínica em estudo. Dessa maneira, poderar-se-ão compreender os aspectos relacionados ao tempo de tratamento, ao tipo (psicoterapia, atendimento psiquiátrico, farmacoterapia) e à natureza da intervenção (terapia cognitivo-comportamental, psicanálise) sob a qual tais pacientes estão submetidos, assim como a definição precisa do diagnóstico psiquiátrico. Além disso, devem ser incluídas outras variáveis pertinentes, que podem estar associadas ao fenômeno investigado (características individuais, de personalidade, familiares, fatores genéticos).

Cabe destacar ainda que os participantes não foram avaliados quanto à presença de possíveis diagnósticos psiquiátricos comórbidos, além daqueles associados ao uso de SPAs. No presente estudo, foram empregados instrumentos de avaliação objetiva das modificações comportamentais e cognitivas relacionadas à ansiedade (BAI) e à depressão (BDI). Contudo, tais medidas não são capazes de estabelecer, de forma isolada, o diagnóstico de depressão e/ou ansiedade. Em pesquisas futuras, é relevante incluir outros instrumentos, como, por exemplo, o Mini International Neuropsychiatric Interview (MINI) (Amorin, 2000) para o diagnóstico preciso de possíveis transtornos mentais comórbidos ao consumo de SPAs.

Por se tratar de um estudo transversal, não foram estabelecidas relações de causa e efeito. Apenas foram evidenciadas associações descritivas, cujos indicadores de causalidade podem estar em pontos ainda não explorados. Delineamentos transversais são úteis para descrever as características de uma população, identificar populações de risco, para programar ações e planejamento em saúde. Ademais, eles possuem baixo custo, podem ser rapidamente implementados e permitem objetividade na coleta de dados. Contudo, apresentam limitações que merecem destaque, como, por exemplo, a dificuldade em pesquisar condições de baixa prevalência, que exigem amostras maiores. Esse tipo de delineamento trabalha, fundamentalmente, com casos prevalentes do desfecho e com a coleta de dados sobre exposição e desfecho em apenas um momento temporal. Por isso, casos com maior duração são mais facilmente incluídos enquanto aqueles problemas de menor duração têm pouca probabilidade de serem demonstrados nos resultados. Consequentemente, em um estudo desta natureza, torna-se difícil discernir se as exposições estudadas se associam ao surgimento de casos recentes ou se elas se vinculam a casos duradouros (viés de prevalência). Além disso, em função da exposição e do desfecho serem coletados concomitantemente, não se pode estabelecer uma relação temporal entre os eventos e considerar, com maior certeza, se a relação entre eles é ou não de causa e efeito (Bastos \& Duquia, 2007).

Embora os resultados obtidos não possam ser generalizados para outras populações, eles contribuem para o melhor entendimento dos fatores associados ao consumo de SPAs em ambulatórios de saúde mental (comorbidades, expectativas positivas em relação aos efeitos do álcool). Por fim, os autores desta pesquisa sugerem que novas investigações sejam implementadas, empregando delineamentos mais sofisticados (longitudinais), que possibilitem a observação do fenômeno estudado ao longo do tempo, tornando, assim, possível dimensionar, apropriadamente, a prevalência de fatores de vulnerabilidade associados ao consumo de SPA em amostras clínicas.

\section{Referências}

Alves, H., Kessler, F., \& Ratto, L. R. C. (2004). Comorbidade: Uso de álcool e outros transtornos psiquiátricos. Revista Brasileira de Psiquiatria, 26(supl. 1), S51-S53. 
Amorin, P. (2000). Mini International Neuropsychiatric Interview (MINI): Validação de entrevista breve para diagnóstico de transtornos mentais. Revista Brasileira de Psiquiatria, 22, 106-115.

Araújo, L. B., \& Gomes, W. B. (1998). Adolescência e as expectativas em relação aos efeitos do álcool. Psicologia: Reflexão e Critica, 11, 5-33.

Bastos, J. L. D., \& Duquia, R. P. (2007). Um dos delineamentos mais empregados em epidemiologia: Estudo transversal. Scientia Médica, 17, 229-232.

Beck, A. T., Epstain, N., Brown, G., \& Steer, R. A. (1988). The Beck anxiety inventory. Journal of Consulting and Clinical Psychology, 56, 893-897.

Beck, A., Ward, C., Mendelson, M., Mock, J., \& Erbaugh,G. (1961). An inventory for measuring depression. Archives of General Psychiatry, 4, 561-571.

Bittencourt, S. M., \& Oliveira, M. S. (2005). Estudo de relações entre fobia social e uso do álcool. Revista Brasileira de Terapias cognitivas, 1(2), 135-144.

Blume, T. W., Green, S., Joanning, H., \& Quinn, W. S. (1994). Social role negotiation skills for substance-abusing adolescents: A group model. Journal of Substance Abuse Treatment, 11, 197-204.

Bongers, I. M., van Oers, H. A., van de Goor, I. A., \& Garretsen, H. F. (1997). Alcohol use and problem drinking: Prevalences in the general Rotterdam population. Substance Use \& Misuse, 32, 1491-1512.

Calheiros, R. V., Oliveira,M.S.,\&Andretta,I.(2006). Comorbidades psiquiátricas no tabagismo. Aletheia, 23, 65-74.

Carlini, E. A., Galduróz, J., Silva, A., Noto, A., Fonseca, A., Carlini, C., Oliveira, L. G., Nappo, S. A., Moura, Y. G., Sanchez, \& Z. V. D. M. (2006). II levantamento domiciliar sobre o uso de drogas psicotrópicas no Brasil: Estudo envolvendo as 108 maiores cidades do país. São Paulo: CEBRID / UNIFESP.

Castel, S., Rush, B., Urbanoski, K., \& Toneatto, T. (2006). Overlap of clusters of psychiatric symptoms among clients of a comprehensive addiction treatment service. Psychology of Addictive Behaviors, 20, 28-35.

Christiansen, B., Smith, G., Roheling, P., \& Goldman, M. (1989). Using alcohol expectancies to predict adolescent drinking behavior after one year. Journal of Consulting and Clinical Psychology, 57, 93-99.

Cozby, P. C. (2003). Métodos de pesquisa em ciências do comportamento. São Paulo: Atlas.

Cunha, J. A. (2001). Manual da versão em português das Escalas Beck. São Paulo: Casa do Psicólogo.

Donovan, D., \& Marlatt,A. (1980). Assesment of expectancies and behaviors associated with alcohol comsuption. Journal of Studies on Alcohol, 41, 1153-1185.

Edwards, G., Marshall, J., \& Cook, C. (1999). O tratamento do alcoolismo. Porto Alegre: Artmed.

Fromme, K., \& D’Amico, E. J. (2000). Measuring adolescent alcohol outcome expectancies. Psychology of Addictive Behaviors, 14, 206-212.
Gaffney, L., Thorpe, K., Young, R., Collet, R., \& Occhipinti, S. (1998). Social skills, expectancies, and drinking in adolescents. Addictive Behaviors, 23, 587-599.

Glassman, A. H., Covey, L. S., Stetner, F., \& Rivelli, S. (2001). Smoking cessation and the course of major depression: A follow-up study. The Lancet, 357, 1929-1932.

Goldman, M. S. (1999). Risk for substance abuse: Memory as a common etiological pathway. Psychological Science, 10, 196-198.

Gorestein, C., \& Andrade, L. (1996). Validation of a portuguese version of the Beck Depression Inventory and the State-Trait Anxiety Inventory in Brazilian subjects. Brazilian Journal of Medical and Biological Research, 29, 453-457.

Gouveia, J. P., Ramalheira, C., Robalo, M. T., Borges, J. C., \& Rocha-Almeida, J. (1996). Inventário de expectativas e crenças pessoais acerca do álcool (IECPA) (versão brasileira). São Paulo: Casa do Psicólogo.

Grant, B. F. (1997). Prevalence and correlates of alcohol use and DSM-IV alcohol dependence in the United States: Results of the National Longitudinal Alcohol Epidemiologic Survey. Journal of Studies on Alcohol, 58, 464-473.

Henrique, I. F. S., De Micheli, D., Lacerda, R. B., Lacerda, L. A., \& Formigoni, M. L. (2004). Validação da versão brasileira do teste de triagem do envolvimento com álcool, cigarro e outras substâncias (ASSIST). Revista da Associação Médica Brasileira, 50, 199-206.

Kessler, F., \& Boni, R. (2006). Consenso de cocaína: Cocaína e depressão. Revista da Associação Brasileira de Psiquiatria, 1, 30-33.

Lewis, B. A., \& O’Neill, H. K. (2000). Alcohol expectancies and social deficits relating to problem drinking among college students. Addictive Behaviors, 25, 295-299.

Maluf, T. P. (2002). Avaliação dos sintomas de depressão e ansiedade em uma amostra de familiares de usuários de drogas que frequentam grupos de orientação familiar em um serviço assistencial para dependentes químicos. Dissertação de mestrado não -publicada, Universidade Federal de São Paulo, São Paulo, SP.

Malbergier, A., \& Oliveira Jr., H. P. O. (2005). Dependência de tabaco e comorbidade psiquiátrica. Revista de Psiquiatria Clínica, 32, 276-282.

Marlatt, G. A., \& Gordon, J. R. (1993). Prevenção da recaída: Estratégia e manutenção no tratamento de comportamentos adictivos. Porto Alegre: Artes Médicas.

Melo, W. V., Oliveira, M. S., \& Ferreira, E. A. (2006). Estágios motivacionais, sintomas de ansiedade e depressão no tratamento do tabagismo. Interação em Psicologia, 10, 91- 99.

Messas, G. P., \& Vallada, H. P. (2004). O papel da genética na dependência do álcool. Revista Brasileira de Psiquiatria, 26(Supl. 1), S154-S158.

Monteiro, M. G. (1997). Comorbidade entre dependência de álcool e depressão: Uma visão geral. Informação Psiquiátrica, 4(13), 143-145. 
Morissette, S., Tull, M., Gulliver, S., Kamholz, B., \& Zimering, R. (2007). Anxiety, anxiety disorders, tobacco use, and nicotine: A critical review of interrelationships. Psychological Bulletin, 132, 245-272.

Munaretti, C. L., \& Terra, M. B. (2007). Transtornos de ansiedade: Um estudo de prevalência e comorbidade com tabagismo em um ambulatório de psiquiatria. Jornal Brasileiro de Psiquiatria, 56, 108-115.

Pedroso, R. S., Oliveira, M. S., Araújo, R. B., Castro M. G., \& Melo W. V. (2006). Expectativas de resultados frente ao uso de álcool, maconha e tabaco. Revista de Psiquiatria do Rio Grande do Sul, 28, 198-206.

O'Hare, T., \& Sherrer, M. (1997). Drinking problems, alcohol expectancies, and drinking contexts in college first offenders. Journal of Alcohol and Drug Education, 43, 31-45.

Oliveira, M. S., Wagner, M. F., Zambom, L. F., \& Calheiros, P. R. (2006). Sintomas depressivos em adolescentes usuários de drogas institucionalizados e não institucionalizados. Revista de Psicologia da UnC, 3(1), 21-29.

Peuker,A. C., Fogaça, J., \& Bizarro, L.A(2006). Expectativas e beber problemático entre universitários. Psicologia: Teoria e Pesquisa, 22, 193-200.

Ratto, L., \& Cordeiro, D. C. (2004). Principais comorbidades psiquiátricas na dependência química. In S. Bordin, N. B. Figlie, \& R. Laranjeira (Orgs.), Aconselhamento em dependência química (pp. 167-186). São Paulo: Roca.

Rondina, R. C., Gorayeb, R., \& Botelho, C. (2003). Relação entre tabagismo e transtornos psiquiátricos. Revista de Psiquiatria Clínica, 30, 221-228.

Ross, H. E., Glaser, F. B., \& Stiasny, S. (1998). Sex differences in the prevalence of psychiatric disorders in patients with alcohol and drug problems. British Journal of Addiction, $83,1179-1192$.

Ana Carolina Peuker é Professora Doutora do curso de Especialização em Terapia Cognitiva e Comportamental da Universidade Federal do Rio Grande do Sul.

Roberta Rosemberg é Especialista em Terapia Cognitiva e Comportamental.

Sílvia Mendes Cunha é doutoranda em Psicologia pelo Programa de Pós-graduação em Psicologia do Instituto de Psicologia da Universidade Federal do Rio Grande do Sul.

Lisiane Bizarro Araujo é Professora Adjunta do Programa de Pós-graduação em Psicologia do Instituto de Psicologia da Universidade Federal do Rio Grande do Sul.

Recebido: 05/01/2009

$1^{a}$ revisão: $28 / 03 / 2009$

$2^{a}$ revisão: $22 / 07 / 2009$

Aceite final: 12/08/2009 